\title{
Study of insulator contamination degree on-line monitoring system based on CAN Bus
}

\author{
HUANG Yunxing, a ${ }^{1,}$ PAN Xingdong ${ }^{1}$ and YUAN Xinxing ${ }^{2}$ \\ ${ }^{1}$ Liuzhou Bureau of EHV Power Transmission Company, China South Power Grid Co., Ltd. 545006, Liuzhou, China \\ ${ }^{2}$ Wuhan Kedio Electric Power Technology Co., Ltd, 430223, Wuhan, China
}

\begin{abstract}
In this paper, the design and realization of an online monitoring system for leakage current through the insulators near substation are presented. The system with the distributed structure mainly consists of computer and on-site monitoring units that are based on DSP. The monitoring units involve realtime sampling the leakage current through the insulators together with environment temperature and humidity, preprocessing the data and sending the useful information to the computer by CAN bus. At the same time, the computer achieves the functions of saving data, displaying the information according to users, and predicting the degree of the insulators dirtiness. Through practical tests, the system is proved to be efficient and robust.
\end{abstract}

Keywords-CAN bus ; insulator ; Leakage current ; on-line monitoring

\section{INTRODUCTION}

Once the contamination on the insulation surface being affected with damp, the soluble substances would dissolve in the water gradually and form a layer of conductive water film on the surface of insulator, resulting in insulator surface resistance decreased and constituting a leakage current through the surface of the insulator [1]. When the leakage current increases, partial discharge will eventually lead to flashover, posing a threat to the safety of electric power production [2].

In the power system, it is the potential of different insulator conductor mechanically interconnected parts, a very crucial role its performance on safety plays the entire transmission system. Especially insulators in addition to running outdoors should have a certain electrical insulation properties and certain mechanical properties of electrical insulation properties and a certain intensity, but also exposed to the atmosphere, and long-term work in the cold and heat, wind and rain, damp, grime and other harsh environments, prone to internal insulator cracks, surface damage, reducing the dielectric strength and flashover failure. Flashover is due to the insulator surface contamination layer reaches saturation and moist, the insulator surface leakage current is growing too large, resulting in flashover insulator surface. With the development of urban and rural areas of industry and agriculture, air pollution increasing year by year, flashover accident involving a wide range, long outage time, large economic losses, serious impact on the power system stability, reliability, safety and economy. Traditional cleaning program abroad insulators way, not only a lot of manpower and material resources, and the vast majority of power outages swept, resulting in large economic losses, and this type of program maintenance, cleaning and can not completely eliminate accidents.

For many years, people on the actual operating conditions of power system high voltage transmission line insulators of the substation post insulator and could not find a safe and effective method to monitor. Because the vast majority of these insulators are exposed to the air, over time, we will continue to accumulate insulator surface grime in the air, when wet will reduce the insulation performance of the insulator, if the insulator surface ESDD reaches a certain level, so in the insulator surface damp conditions, the effective surface of the insulator creepage distance will be greatly reduced, the surface of the insulator partial discharge intensity will increase, which occurred in contamination flashover, resulting in the whole and the entire power transmission line failure, paralyzed status, which will bring incalculable harmful consequences to people's lives.

Leakage current is an instantaneous dynamic index determinated by the actual creep age distance, the weather and the degree of contamination. Leakage current is relatively easy to measure, suitable for online detection, it contains enough information about the running state of the insulator, therefore, and we evaluate the running state by measuring the leakage current in the present monitoring system.

This paper constructs a distributed on-line filth insulator monitoring system based on CAN bus, making it to collect the information of the insulator leakage current, environment temperature and humidity throughout the day, using a microcomputer to deal with the feedback information of online monitoring makes the staff understand the insulator filthy state information in time and provides a scientific basis for the transition from planned maintenance to state overhaul.

\section{STRUCTURE DESIGN OF ON-LINE INSULATOR CONTAMINATION MONITORING SYSTEM}

This faulty insulator detecting device with electric field method is the basic principle, its core is based on the head of the optical electric field sensor to measure electric field. Light sources into the single-mode fiber laser coupling, sensing head inside through coupling the laser collimating lens to the space, with the partial linear polarized makes it, after a quarter wave plate again divided into circularly polarized light, the fast axis parallel to the electro-optic crystal respectively under the effect of electric field of two induction spindle, through the crystal, the polarization is changeless, but phase changed, and is related to the measured field intensity. With partial detector 
two perpendicular polarized light beam to extract the same component, get interference light intensity. The emergent light for photoelectric conversion, the change of light intensity changes into electrical signals, reflects the change of the electric field to be tested.

This structure(Figure.1) makes an comprehensive application of some mature technologies such as signal processing, communications, DSP, database, computer network and so on, mainly aimed at realizing online filthydegree monitoring in and nearby the insulator; considering the strong interference、 the operational safety of high voltage line and the module power output of the online monitoring unit, we choose the combination of wired and wireless communications and use HAC- $\mu \mathrm{P}$ wireless low-power module and CAN bus to implement it [6].

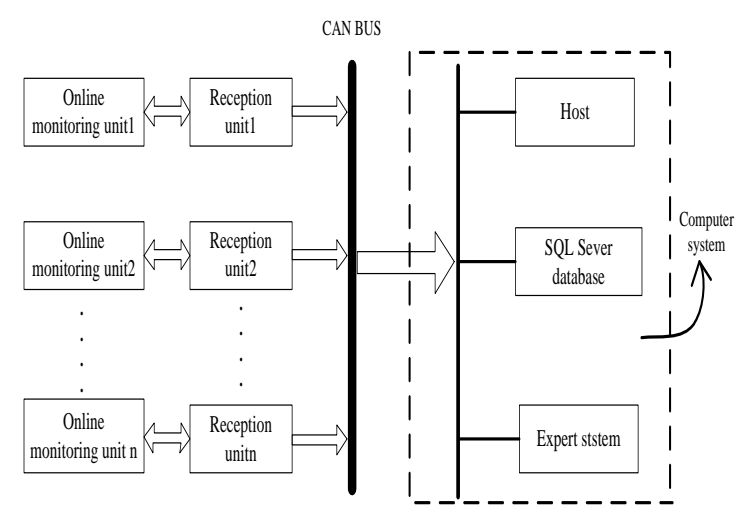

Figure 1. Online monitoring system structure

The online monitoring unit is mainly responsible for realtime acquisition parameters such as the leakage current of insulator string and outside temperature and humidity, using wavelet transform to filter the leakage current, so as to obtain the raw data from reflected signals. Then process the data according to relevant algorithm and extract some identifying information within a certain time interval, such as the maximum and average number of pulses over leakage current value, harmonic average of three and five times, and on the basis of the design of communication protocol in advance, through combining wireless communication and CAN bus communication sent real-time information after pretreatment to the computer system ([7] [8]), realizing the functions such as data storage, display and warning; In addition, the online monitoring unit receives and explains all kinds of operation commands from the computer (real-time waveform request set, normal communication test, setting characteristic information from leakage current to extract time interval, etc.), and through the embedded operating system kernel to adjust the operation relationship between each task. At the same time, the staff with the aid of computer and specialized software can easily understand the filth degree of each running insulator string.

\section{COMMUNICATION PROTOCOL DESIGN}

Many kinds of fieldbus technology exist such as Lon Works, CAN Field Bus, HART and PROFIBUS and so on. Because of its unique design, high reliability, strong anti-jamming capability, flexible structure, low cost, CAN bus is widely used as the interconnection in industrial equipment and is recognized as one of the most promising fieldbus. CAN bus belongs to serial communication bus network, and its main features are: (1) More main work modes, any node on the internet can take initiative to exchange information with the other nodes regardless of master-slave; (2) Unique arbitration technology, real-time performance is higher than CSMA/CD and token ring network; (3) The highest transmission rate is 1 $\mathrm{MB} / \mathrm{s}$ (communication distance is $40 \mathrm{~m}$ ), the farthest distance $10 \mathrm{~km}$ (rate is below $5 \mathrm{~KB} / \mathrm{s}$ ); (4) Using twisted pair as a communication medium has many advantages such as simple interface, easy installation, low networking cost.

Front-end machine is the core of the monitoring system, the structure and quality of front-end hardware circuit is directly related to the success of the leakage current signal processing correctness and the whole monitoring system or not, but also with the front-end software development is closely related to the underlying machine. In this system, the front-end machine is mainly by the sensor electronics module, programmable gain amplifier module, a signal processing module, AD converter keeps module, serial communication module, DA conversion output modules, communication modules, automatic reset module, solar power system module etc.

As shown in Figure 2, the underground receivers mainly complete the function of combining wireless communication with wired communication, on one hand, they pass on the received wireless data to computer through CAN bus; on the other hand, they sent commands from the computer to the online monitoring unit through wireless module, so as to realize the communication between the on-line monitoring unit and the computer.

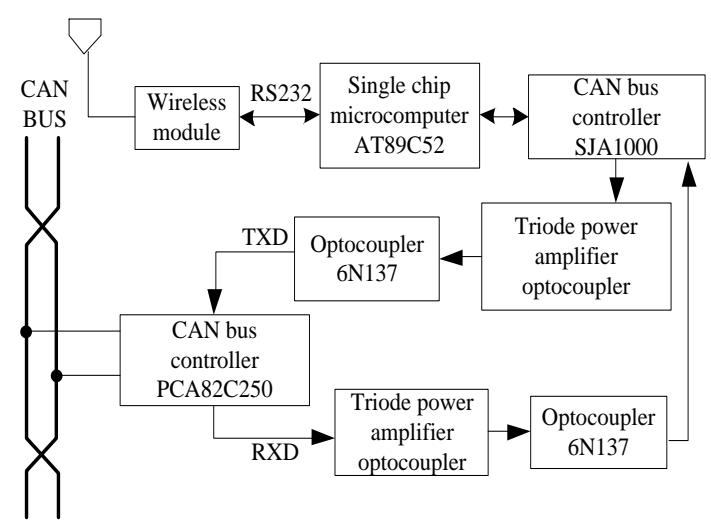

Figure 2. Ground reception unit structure

In order to guarantee the reliable communication, the system has been designed a simple communication protocol, 
the frame structure of the project is: Oxaa, Ox55, destination address, source address, data length, command word, effective data, efficacy, Ox3c, Oxc3. Frame head and tail are marked by two bytes coming from the combination of positive code and inverse code, and keep $0 \mathrm{~s}$ and $1 \mathrm{~s}$ equal in number within 1 byte, this kind of design can minimize imbalance of code stream causing by the large difference between $0 \mathrm{~s}$ and $1 \mathrm{~s}$ and reinforce the test results of frame head. The transmit of protocol packets include sending, waiting for the other confirmation, confirmation or delaying for resending.

\section{EQUATIONS AND MATHEMATICS}

\section{A. Structure diagram of online monitoring unit and working principle}

As shown in figure 3, online monitoring unit uses DSP TMS320LF2407 as the core and equips with C/OS II embedded operating system software, in order to realize the reasonable scheduling between tasks, satisfying the system requirement of real-time performance. The DSP chip can process data at a high speed and integrate up to 16 high-speed A/D analog input channels and rich peripheral circuits, simplifying the system design and improving the system performance.

The concrete working principle for online monitoring unit is: the lower humidity and lighter filthy, the smaller leakage current amplitude is ( $\ngtr 1 \mathrm{~mA}$ ), while the bigger leakage current amplitude of flash or line overvoltage conditions is $(>10 \mathrm{~mA})$. Therefore, in order to adapt to the widespread change of current and requirement of real time, at the same time improve the signal-to-noise ratio of the sampling circuit, the system adopts double branch current measurement circuit which means that the leakage current is converted into the voltage signal firstly through the current sensor, and then divided into two roads running into different signal conditioning circuits at the same time, signal sampling circuit collects both roads signals and selects the data after judging the ranges ,so as to realize the accurate measurement of the leakage current.

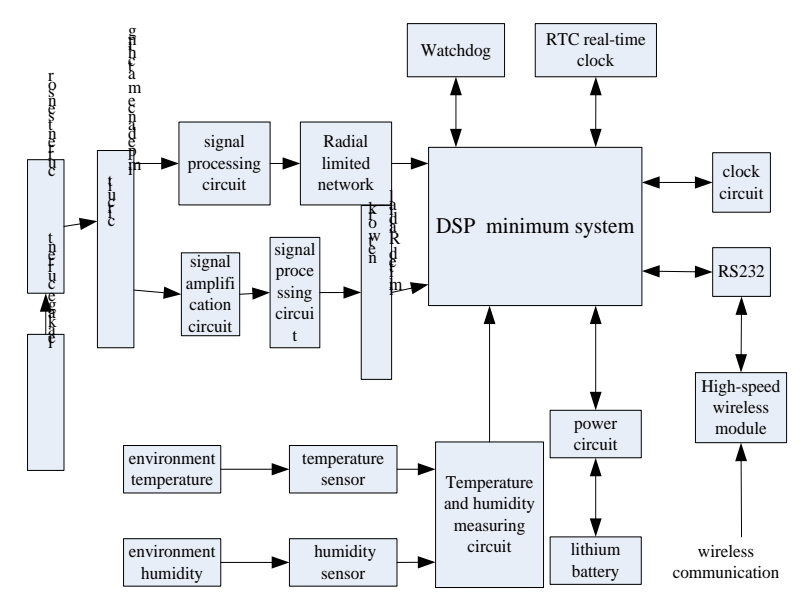

Figure 3. Online monitoring unit structure diagram
Control circuit of the main role is to control the action of testing equipment, as well as the subsequent information processing, mainly including start detection device, control the movement of the actuator, receive insulator electric field testing data, packaging required data detection, location, time, etc., and related parts. Controller hardware structure function as shown in the figure below:

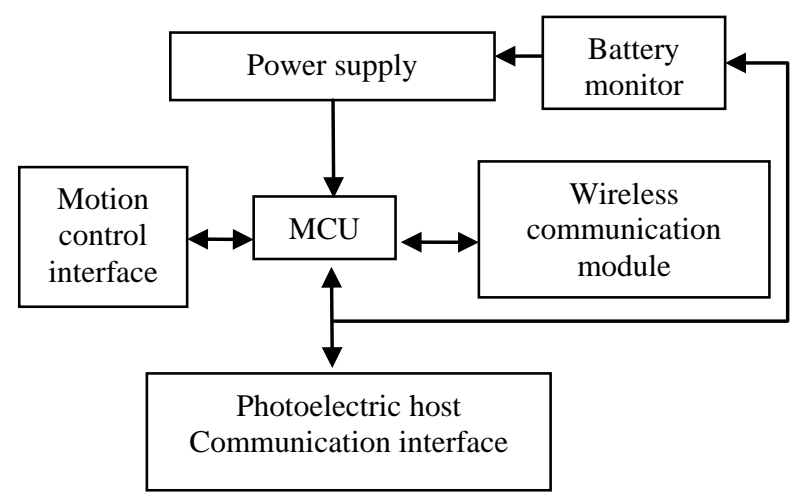

Figure.4 Functional Diagram

Communication control section and the main body of the robot using wireless transmission, convenient on-site communications and operations staff; insulator strings will be measured to detect data transmission to the next tower computers and remote control for field staff to get real-time observation Inspection results; income data can be stored and played back in real time, so you can easily check the entire tower insulator string data; real-time comparison of normal insulator string electric field distribution curves were analyzed to determine the position of the low value of zero insulator and displayed in real time to determine the results.

\section{B. Design of online monitoring unit software}

To improve the software reliability and speed up the development, the online monitoring unit uses $\mu \mathrm{C} / \mathrm{OS}$ II ERTOS real-time embedded operating system as the basis and the support of the system software, scheduling individual tasks to realize real-time parallel, orderly, reliable operation which provides guarantee for the stable operation of the system. The specific software in the design launches the modulus conversion by period interrupting event using timing 3, in order to make A/D sample at a fixed period, set the INTPRI in the ADC registers from 1 to 0 and make the $A / D$ interrupt in high priority mode, which makes its priority higher than that of serial communication interrupt and operating system clock source, in order to realize the real-time sampling of current signal. At the same time double buffer method is used to avoid covering the data in processing area during the real-time sampling, one interrupting service routine (ISR) as shown in figure 4; General timer 1 is used as the clock source in the operating system and its ticks is set to $200 \mathrm{~Hz}$, which means the system makes clock interrupt every $5 \mathrm{~ms}$ so that the operating system can carry on the task scheduling. 


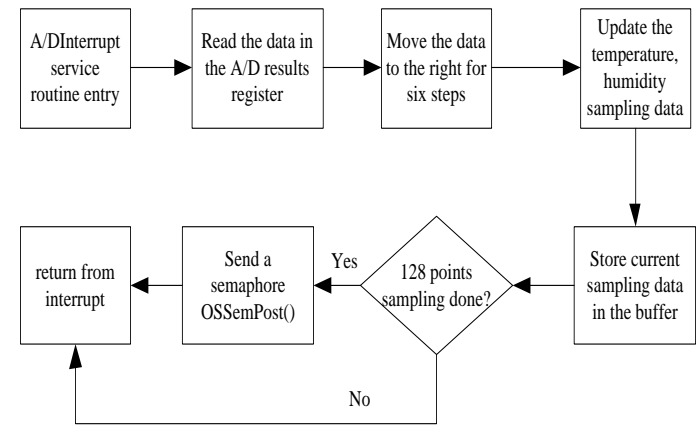

a) Figure 5. Current sampling interrupt service routine

\section{Low consumption and anti-interference function design of online monitoring unit}

The monitoring of online monitoring unit is not uninterrupted, because the battery and wireless communication flow is very difficult to achieve such a request. It is therefore imperativly to design a sleep mode, that is the staff does not need it to monitor and it has been automatically entered into a dormant state when the online monitor ends sending data to the host computer. The related things work after the scheduled time reaches or staff sends command instruction. The principle of low power design is in dormancy for power control of quipment outside of the main controller in sleep. Lithium batteries supplies power for SCM by voltage chip, and power sensors and communication modules is accomplished by VCCL chip. The timing function when the SCM sleep can be realized by use of $32.768 \mathrm{kHz}$ external SCM crystal and combining with built-in timer. The main controller switches from sleep to work state by the interrupt caused by overflow of the timer.

The transmission line holds high voltage, transmission current and long line and will produce strong electromagnetic field within its power corridor. As a result of the transmission line corona effect and accompanied by a lot of noise, it causes error of sensors, unnormally operation of SCM, communications received interference and other problems. The following measures can be used to enhance to enhance the system's ability to resist electromagnetic radiation:

1) Electronic devices should be chosen higher integration and stronger anti-interference components strictly. It needs initialized correction and rational distribution for each device to ensure that the system has strong electromagnetic compatibility.

2) The hardware anti-interference technology can be used. The fully considering the importance of parameters when selecting device can effectively suppress interference sources and remove most interference. The common hardware antiinterference techniques are: shielding, grounding, decoupling technology and isolation technology.

3)The software anti-interference technology. The common software anti-interference techniques are: digital filtering technology, automatic calibration technology when transmission information and self-recovery technology when failure occurs. It has the advantages of low cost, strong flexibility and the concise method.
4) Some anti-interference measures have to take in the subsequent software design. For example, it is easy to cause the program counter pointer value change when subjected high interference destroying the normal operation. Aiming at the problem, empty operation instruction is added at the key program statements and built-in watchdog timer of the main controller is enable to prevent the program run fly or crash and so on.

\section{CONCLUSION}

The on-line monitoring system based on CAN bus designed in this paper can forecast the filth degree of the running insulator; online monitoring unit uses DSP TMS320LF2407 as the core and equips with C/OS II embedded operating system software to improve the real-time performance and reliability of the system. The good application of the system can not only improve the reliability of overall power system , provide conditions for the transition from planned maintenance to state overhaul for the electric power department, but also provide a feasible method for the study of pollution flashover experiments. After reasonable object-oriented analysis for simulation program, Variables and operations in program can easily be stored in different categories. Package features greatly enhance the security of variables, so we don't worry variables will be misused or duplicate definitions. The development of high performance and ease of installation leakage current through the core type sensor that uses card-mounted structure can be implemented without changing the installed coupling structure tower insulators and the premise is very easy to live action. Unlike using a conventional integrating circuit through the core type sensor adapter circuit, namely the installation of the adaptation circuit of Rogowski coil, improve the wear performance of core type sensor to solve the traditional low sensor accuracy through the core, narrow band of the core material parameters sensitive features

\section{References}

[1] Tao Yuanzhong, Bao Jianqiang, transmission line insulator running technology handbook. Beijing: China power press (2003)

[2] Wang Jun, $\mathrm{Xu}$ Hongchun, the reflection of the pollution flashover accident, rural electrification, (6): 29 (2003)

[3] Nie Yixiong, Yin Xianggen, the study of on-line insulator detection method. Electric porcelain arrester, (2): 3-8(2000)

[4] Huang Yinbo, Liu Gubing, Wang Xiangli, etc., the insulator filthy online telemetry system based on GPRS network transmission line. Automation of electric power systems, 28 (21): 92-95 (2004)

[5] Shi Yonghui, Li Dehua, Cai Tao, study of high voltage insulator on-line monitoring and fault location system High voltage technology, 29 (11) 23-25 (2003)

[6] Tong Xiaoyang, Zhang Guangchun, Zou Siyi, the design and implementation of distributed insulation online monitoring system based on CAN bus. Automation of electric power systems, 21 (3) : 57-60 (2013)

[7] Rao Yuntao, Zou Jijun, Zheng Yongyun, fieldbus CAN principle and application technology. Beijing: Beijing University of aeronautics and astronautics press,(2003)

[8] Liao Shenglan, Xie Shuanqin, reactive power compensation system based on CAN bus design. Journal of low voltage apparatus, (6): 34-36 (2014) 